\title{
AtPIG-S, a predicted Glycosylphosphatidylinositol Transamidase subunit, is critical for pollen tube growth in Arabidopsis
}

Nicholas Desnoyer ${ }^{1,2}$, Gregory Howard ${ }^{1}$, Emma Jong ${ }^{1}$ and Ravishankar Palanivelu ${ }^{1 *}$ (I)

\begin{abstract}
Background: Glycosylphosphatidylinositol (GPI) addition is one of the several post-translational modifications to proteins that increase their affinity for membranes. In eukaryotes, the GPI transamidase complex (GPI-T) catalyzes the attachment of pre-assembled GPI anchors to GPI-anchored proteins (GAPs) through a transamidation reaction. A mutation in AtGPI8 (gpi8-2), the putative catalytic subunit of GPI-T in Arabidopsis, is transmitted normally through the female gametophyte (FG), indicating the FG tolerates loss of GPI transamidation. In contrast, gpi8-2 almost completely abolishes male gametophyte (MG) function. Still, the unexpected finding that gpi8-2 FGs function normally requires further investigation. Additionally, specific developmental defects in the MG caused by loss of GPI transamidation remain poorly characterized.
\end{abstract}

Results: Here we investigated the effect of loss of AtPIG-S, another GPI-T subunit, in both gametophytes. Like gpi82, we showed that a mutation in AtPIG-S (pigs-1) disrupted synergid localization of LORELEI (LRE), a putative GAP critical for pollen tube reception by the FG. Still, pigs-1 is transmitted normally through the FG. Conversely, pigs-1 severely impaired male gametophyte $(\mathrm{MG})$ function during pollen tube emergence and growth in the pistil. A pPIGS:GFP-PIGS transgene complemented these MG defects and enabled generation of pigs-1/pigs-1 seedlings. However, the PPIGS:GFP-PIGS transgene seemingly failed to rescue the function of AtPIG-S in the sporophyte, as pigs-1/pigs-1, pPIGS:GFP-PIGS seedlings died soon after germination.

Conclusions: Characterization of pigs-1 provided further evidence that the FG tolerates loss of GPI transamidation more than the MG and that the MG compared to the FG may be a better haploid system to study the role of GPIanchoring. Pigs-1 pollen develops normally and thus represent a tool in which GPI anchor biosynthesis and transamidation of GAPs have been uncoupled, offering a potential way to study free GPI in plant development. While previously reported male fertility defects of GPI biosynthesis mutants could have been due either to loss of GPI or GAPs lacking the GPI anchor, our results clarified that the loss of mature GAPs underlie male fertility defects of GPI-deficient pollen grains, as pigs-1 is defective only in the downstream transamidation step.

Keywords: Glycosylphosphatidylinositol, Transamidase complex, Pollen, Pollen tube, Female gametophyte, Synergids, Seedling lethal

\footnotetext{
* Correspondence: rpalaniv@email.arizona.edu

${ }^{1}$ School of Plant Sciences, University of Arizona, Tucson, AZ 85721, USA

Full list of author information is available at the end of the article
}

(C) The Author(s). 2020 Open Access This article is licensed under a Creative Commons Attribution 4.0 International License, which permits use, sharing, adaptation, distribution and reproduction in any medium or format, as long as you give appropriate credit to the original author(s) and the source, provide a link to the Creative Commons licence, and indicate if changes were made. The images or other third party material in this article are included in the article's Creative Commons licence, unless indicated otherwise in a credit line to the material. If material is not included in the article's Creative Commons licence and your intended use is not permitted by statutory regulation or exceeds the permitted use, you will need to obtain permission directly from the copyright holder. To view a copy of this licence, visit http://creativecommons.org/licenses/by/4.0/. The Creative Commons Public Domain Dedication waiver (http://creativecommons.org/publicdomain/zero/1.0/) applies to the data made available in this article, unless otherwise stated in a credit line to the data. 


\section{Background}

In Arabidopsis thaliana (Arabidopsis) plant reproduction, pollen grains land on the stigma of a flower, adhere to the papillae of the stigma, and signal them to initiate hydration and germination of the compatible pollen grain [26, 41]. Upon germination, a pollen tube will extend through the style and transmitting tract of the pistil and emerge into an ovule chamber, where it targets a female gametophyte (FG) and completes double fertilization. The two synergid cells (synergids) found at the micropylar end of the FG are transfer cells, which are specialized in secretion of factors for proper communication with and reception of the pollen tube [18].

Synergids develop a region of highly dense cell wall and plasma membrane invaginations at their micropylar end called the filiform apparatus (FA) that is shared by both synergids. Extensive plasma membrane invaginations in the FA increases the total surface area of the synergids, promoting secretion of proteins and molecules out of the cell and generating an extracellular microenvironment conducive to attraction [14] and reception [37] of pollen tubes. After entering the ovary chamber, pollen tubes respond to the chemoattractants from the synergid cells, migrate steadfastly to an ovule, enter the micropylar opening in the ovule, and reach the FA of the synergid cells, the first physical point of contact between the pollen tube and the synergid cells [20]. The cessation of pollen tube growth in one of the synergids (receptive synergid cell) and subsequent release of sperm cells to induce double fertilization is collectively called pollen tube reception, a process that is regulated by genes expressed in both gametophytes [20].

In the FG, LORELEI (LRE), a predicted glycosylphosphatidylinositol (GPI)-anchored protein (GAP), binds the receptor-like kinase FERONIA (FER) to form a coreceptor complex critical for the perception of an unknown ligand to transduce downstream signaling necessary for pollen tube reception [24, 25]. Homozygous lre-7/lre-7 [39], fer-4 mutant [5], and lre-7, fer-4 double mutant pistils [25] all show similar levels ( $~ 80 \%)$ of reduction in seed set, indicating both proteins function together in the same pathway. Using a pLRE:LRE-cYFP translational fusion construct, we previously showed that the seed set defect in lre mutants can be fully rescued and that LRE-cYFP localizes in the FA of synergid cells [25]. Roughly $68 \%$ of all cYFP signal in the synergids expressing LRE-cYFP accumulate in the FA. Deletion of both predicted omega sites in LRE, residues to which GPI is expected to be post-translationally attached, or complete ablation of the GPI attachment signal (GAS) region in the C-terminus of LRE protein, dramatically disrupts the localization of LRE-cYFP in the FA. While this disruption in localization of these mutant LRE-cYFP is suggestive of their failure to receive a GPI anchor, both mutants fully complement pollen tube reception and seed set defects in lre-7/lre-7 [25]. Even replacing the GAS region in LRE, with the transmembrane domain of FER, fully complements lre-7 female fertility defects, showing that LRE can embed in the plasma membrane in a completely different form and still function in pollen tube reception to near normal levels [25].

To address whether the loss of GPI anchor addition to other FG-expressed GAPs is tolerated, in addition to the cis-mutations in LRE, we previously also analyzed a mutation in a subunit of the GPI transamidase (GPI-T), the transacting complex responsible for GPI anchor addition to GAPs by transamidation [25]. A T-DNA insertion in GPI8 (gpi8-2), the putative catalytic subunit of GPI-T, affected the polar localization of LRE-cYFP in the FA of synergids at a similar level to the cis-mutations in GAS region of LRE. Still, pollen tube reception and seed set in gpi8-2/+ pistils was indistinguishable from wild-type pistils, and near normal gpi8-2 transmission through the FG suggested that loss of GPI anchor addition to GAPs in the FG does not disrupt its function. In contrast, transmission of gpi8-2 through the MG was abolished and gpi8-2 homozygotes could not be established [25]. The precise MG function that is affected in gpi8-2 and confirmation of AtGPI8 function in MG by complementation assays have not been reported. Consequently, which aspect of pollen function in fertilization (pollen development, pollen tube emergence, pollen tube growth, and pollen tube reception) is disrupted from loss of GPI anchor addition to GAPs expressed in the MG remains unknown.

The normal function of gpi8-2 FGs was rather unexpected, as GPI anchor addition to GAPs is the most complex and metabolically costly lipid post-translational modification in eukaryotes [32] and is most likely used as a mechanism for the membrane attachment of many other GAPs in FG function. To obtain additional evidence on how GPI-T loss affects FG and MG in this study, we investigated the defects in Arabidopsis FG and MG caused by a mutation in AtPIG-S (At3g07180), a homolog of yeast and human PIG-S and a putative subunit of GPI-T. In addition, we utilized the reporter gene in the T-DNA that disrupted AtPIG-S to pinpoint specific MG phenotypes caused by the loss of AtPIG-S in GPI-T.

The GPI-T complex consists of 5 subunits - PIG-K/ GPI8, GPAA1/GAA1, PIG-S/GPI17, PIG-T/GPI16, and PIG-U/GAB1 - in mammals and yeast, respectively [9]. Arabidopsis homologs of each of these GPI-T subunits have been identified [6] and is comprised of 5 subunits PIG-K/GPI8 (At1g08750), GAA1 (At5g19130), PIG-S (At3g07180), PIG-T (At3g07140), and PIG-U (At1g63110) (Luschnig \& Seifert 2011). Of these, only the catalytic GPI8 subunit had been characterized in greater detail in 
Arabidopsis [2, 25]. In this study, we showed that a mutation in AtPIG-S, a putative GPI-T subunit in Arabidopsis, does not affect the function of the FG even though it disrupts localization of LRE-cYFP. Furthermore, our results demonstrated that pigs- 1 transmission through the MG is almost completely abolished due to defects at the stages of pollen tube emergence and tube growth, revealing the importance of GPI anchor addition to the MG-expressed GAPs during pollen pistil-interactions. Characterization of the pigs-1 mutation also showed that GPI transamidationdeficient pollen grains develop normally and thus represent a tool in which GPI anchor biosynthesis and GPI anchor addition to GAPs have been uncoupled, offering a potential way to study of free GPI in plant development. Restoration of normal tube growth in pigs-1 pollen by supplying a wildtype copy of the AtPIG-S fused to GFP and expressed from its own promoter ( $p$ PIGS:GFP-PIGS) allowed the generation of pigs-1/pigs-1 seeds. Our results reported in this study using pigs-1 are consistent with previous results obtained using gpi8-2 and showed that the FG is far more tolerant than the MG to the loss of GPI-T function and GPI anchor addition to GAPs.

\section{Results}

A mutation in AtPIG-S disrupted localization of LORELEI in the filiform apparatus of synergid cells

To examine if loss of GPI-T subunits (and by extension, loss of GPI anchoring) disrupts function of the Arabidopsis FG, we obtained a T-DNA insertion in AtPIG-T (pigt-1). PIG-T is the central component of GPI-T and is critical for the formation of complex by binding the luminal domains of GAA1, the largest subunit of GPI-T that likely presents GPI to the complex reaction center, and PIG-S, a peripheral component with unknown function [31]. As was done with gpi8-2 [25], we used disruption of LRE-cYFP localization in the FA of synergids of the pigt-1 mutant as a marker to characterize AtPIG-T function in the FG. Surprisingly, all ovules in pigt-1/+, pLRE:LRE-cYFP/ pLRE:LRE-cYFP pistils showed a localization pattern that is indistinguishable from LREcYFP localization in wild-type background (Additional file 1), despite AtPIG-T being predicted to encode a functionally important subunit of GPI-T [31]. RT-PCR and RT-qPCR experiments using 14-day old homozygous pigt-1/pigt-1 seedlings showed that full-length AtPIG-T transcripts were not detected in pigt-1 seedlings and that truncated AtPIG-T mRNA transcripts, consisting of exons 1-4, accumulated to a level that is only $40 \%$ compared to wild type (Additional file 2). Based on these results we concluded that AtPIG-T transcripts lacking exon 5, which encodes the cytosolic domain and ERretrieval motif of PIG-T [7], coupled with the lower levels of these partial transcripts, is still not sufficient to cause a noticeable reduction in LRE-cYFP localization in the FA of pigt-1 synergids. Consequenlty, we did not use pigt-1 any further to investigate if disrupting GPI-T function affects Arabidopsis FG functions.

We next obtained a T-DNA mutant line (pigs-1) in AtPIG-S, another putative subunit of GPI-T. Homozygous pigs-1/pigs-1 could not be obtained (Table 1), so we evaluated if pigs-1 disrupts LRE localization in the FA by analyzing localization of LRE-cYFP in pistils heterozygous for pigs-1 and homozygous for pLRE:LREcYFP (Fig. 1). We detected two patterns of LRE-cYFP localization in pigs-1/+ pistils. In one portion of ovules $(\sim 60 \%, n=334$; Fig. 1e), we detected expected LREcYFP localization in the FA and in puncta in the synergid cytoplasm (Fig. 1f), as reported previously [25]. In the remaining ovules $(\sim 40 \%, n=334$; Fig. 1e), LREcYFP localization was aberrantly diffused throughout the synergids (Fig. 1g). The two types of localization pattern observed here were similar to ovules in heterozygous gpi8-2 pistils that we previously reported [25]. Under similar assay conditions, almost $100 \%$ of ovules in wildtype pistils carrying the $p L R E-c Y F P$ transgene had the expected polarized LRE-cYFP localization pattern in synergids ( $n=229$; Fig. 1e). When averaging the relative integrated signal density within the FA of ovules with diffused localization in a pigs-1/+ pistil, they showed about a $50 \%$ reduction in signal in the FA and had substantially more signal dispersed towards the chalazal end of the synergid cell compared to that in sibling ovules with a polarized LRE-cYFP localization in the FA (Figs. $1 \mathrm{~h}$ and i). These results showed that AtPIG-S is important for polarized localization of LRE in the FA, likely by mediating GPI anchor addition to LRE-cYFP.

\section{Transmission of pigs- 1 is primarily affected in the male gametophyte owing to defects in pollen tube emergence and growth}

Disruption of LRE-cYFP localization in the FA of 40\% of ovules in a pigs-1/+ pistil coupled with our inability to identify a pigs-1 homozygote suggested that AtPIG-S may be affecting GPI anchor addition to LRE and other synergid-expressed GAPs in the FG. Additionally, it could also play an important role in the MG. We therefore investigated the role of AtPIG-S in either of the two gametophytes by calculating the transmission efficiency (TE) of the pigs-1 mutation through the gamteophytes using the basta resistance marker linked to the pigs-1 $\mathrm{T}$ DNA (Table 1 and Fig. 2a).

Despite the disrupted LRE-cYFP localization in pigs-1+ pistils, the segregation ratio of basta-resistant seeds collected from pigs-1/+ pistils that were pollinated with wild-type pollen indicated that there is normal TE of pigs-1 through the FG (95\%; Table 1). In contrast, only $2 \%$ of seeds collected from wild-type pistils pollinated with a pigs-1/+ pollen were basta-resistant, revealing an 
Table 1 Reduced transmission of the pigs-1 and gpi8-2 mutations through the male gametophyte

\begin{tabular}{|c|c|c|c|c|c|c|}
\hline \multirow{2}{*}{$\begin{array}{l}\text { Female } \\
\text { parent }\end{array}$} & \multirow[t]{2}{*}{ Male parent } & \multicolumn{2}{|c|}{ Observed No. of progeny } & \multirow{2}{*}{$\begin{array}{l}\text { TE } \\
(\mathrm{R} / \\
\mathrm{S})\end{array}$} & \multirow[t]{2}{*}{$x^{2}$} & \multirow[t]{2}{*}{$P$-value } \\
\hline & & Basta $^{\text {Ra }}$ & Basta $^{\mathrm{Sa}}$ & & & \\
\hline BastaR/+ & Wild type & 120 & 131 & 0.92 & 0.48 & 0.49 \\
\hline $\mid r e-7 /+$ & Wild type & 29 & 181 & 0.16 & 110 & $<0.001$ \\
\hline gpis-2/+ & Wild type & 177 & 169 & 1.05 & 0.19 & 0.67 \\
\hline pigs-1/+ & Wild type & 255 & 268 & 0.95 & 0.32 & 0.57 \\
\hline Wild type & BastaR/+ & 189 & 193 & 0.98 & 0.04 & 0.84 \\
\hline Wild type & gpi8-2/+ & 0 & 607 & 0 & 607 & $<0.001$ \\
\hline Wild type & pigs- $1 /+$ & 8 & 483 & 0.02 & 460 & $<0.001$ \\
\hline Wild type & pigs-1/+; GFP-PIGS & 108 & 142 & 0.76 & 4.62 & 0.03 \\
\hline
\end{tabular}

Wild type, Columbia-0 ecotype

$\mathrm{TE}$, Transmission efficiency was calculated as the quotient of the number of basta resistant (R) divided by basta susceptible (S) progeny of the indicated cross X2 was calculated based on the expectation of a 1:1 segregation of basta resistance to susceptibility in the progeny. GFP-PIGS refers to the T2 line of pPIGS:GFP-PIGS \#15-3

${ }^{\mathrm{a} B a s t a}$ resistant $\left(\mathrm{Basta}^{\mathrm{R}}\right)$ and Basta susceptible $\left(\right.$ Basta $\left.^{\mathrm{S}}\right)$ progeny. Basta resistance gene is linked with the T-DNA that is inserted into the AtGPI8 (gpi8-2) [25] and LRE (Ire-7), AtPIGS (pigs-1), and in an unknown location in the genome of the BastaR/+ line [21]

almost complete abolishment of pigs-1 transmission through the MG (Table 1). Furthermore, the transmission of pigs-1 through the MG could not be restored by limited pollination of pigs-1/+ pollen on wild-type pistils (Table 2), indicating that the decreased transmission of pigs-1 through the MG is not attributable to mutant pollen being outcompeted by wild-type pollen; instead, these results point to inherent defects in pigs-1 pollen function.

To identify the defect underlying the reduced transmission of pigs-1 through the male gametophyte, we utilized the pLAT52:GUS gene in the pigs-1 T-DNA as a reporter to assess pollen development and function (Fig. $2 \mathrm{a})$. The pigs-1 mutation is in a quartet/quartet background that prevents microspores from separating even after pollen maturation [34], allowing direct comparison of the wild-type and mutant pollen in each tetrad. Indehiscent and dehiscent pollen tetrads from pigs-1/+ plants were stained for GUS activity $(n=100)$ and in both instances, pigs-1 pollen grains (GUS-positive) had a morphology that is indistinguishable from the wild-type pollen (GUS-negative) on the same tetrad or all pollen grains in the control line (pLAT52:GUS/+ tetrads without any pigs-1 mutation) (Figs. 2b-e), indicating normal development of pigs-1 pollen grains. In addition, pigs-1 pollen grains expressed GUS similar to pLAT52:GUS pollen tetrads in wild-type background, indicating that mature pigs- 1 pollen grains are metabolically active and viable.

When pigs-1/+ pollen grains were crossed to wild-type pistils, the pigs-1 pollen showed dramatic pollen tube growth defects. Unlike wild-type pistils pollinated with pollen carrying pLAT52:GUS in the wild-type background (Fig. 2h), wild-type pistils pollinated with pigs$1 /+$ pollen showed many non-germinated or barely emerged pigs-1 pollen tubes (Figs. $2 \mathrm{f}$ and I and Additional file 3). Many pigs-1 pollen grains remained on the stigma despite GUS staining procedure and mounting onto slides, suggesting that these pollen grains had likely successfully adhered to the stigma papillae, a critical first step in compatible pollen-pistil interactions [20]. Of the pollen grains that germinated pollen tubes, the average longest pigs-1 pollen tube in these pistils was $0.38 \mathrm{~mm} \pm$ $0.09(n=5)$, whereas wild-type pLAT52:GUS pollen tubes grew the entire length of pistils with an average longest length of $2.13 \mathrm{~mm} \pm 0.15(n=5)$. While mutant pigs-1 pollen defects primarily manifest at the stage of pollen tube emergence and growth, in one rarely observed instance of a pigs-1 pollen tube growing along the funiculus of an ovule, disrupted micropylar guidance was observed (Fig. 2g). Based on these results, we concluded that pigs-1 pollen is defective in pollen tube emergence and growth and that these defects underlie the nearly abolished transmission of pigs-1 mutation through the pollen.

\section{The pPIGS:GFP-PIGS transgene rescued pollen tube emergence and growth defects in pigs-1 pollen}

To confirm that the observed MG defects in pigs-1 pollen are solely due to disruption of the AtPIG-S gene, we generated a pPIGS:GFP-PIGS translational fusion construct in which the $1500 \mathrm{bp}$ upstream sequence of the AtPIG-S transcription start site drove the expression of GFP fused to the N-terminus of the AtPIG-S genomic sequence (Additional file 4a). Unfortunately, PIGS-GFP signal was not detectable in seedlings or pollen grains of T1 plants similar to a previously reported GPI-T subunit fusion construct, AtGPI8-EGFP [2]. To verify if the pPIGS:GFP-PIGS is expressed, we infiltrated Agrobacterium carrying the pPIGS:GFP-PIGS construct into $N$. 

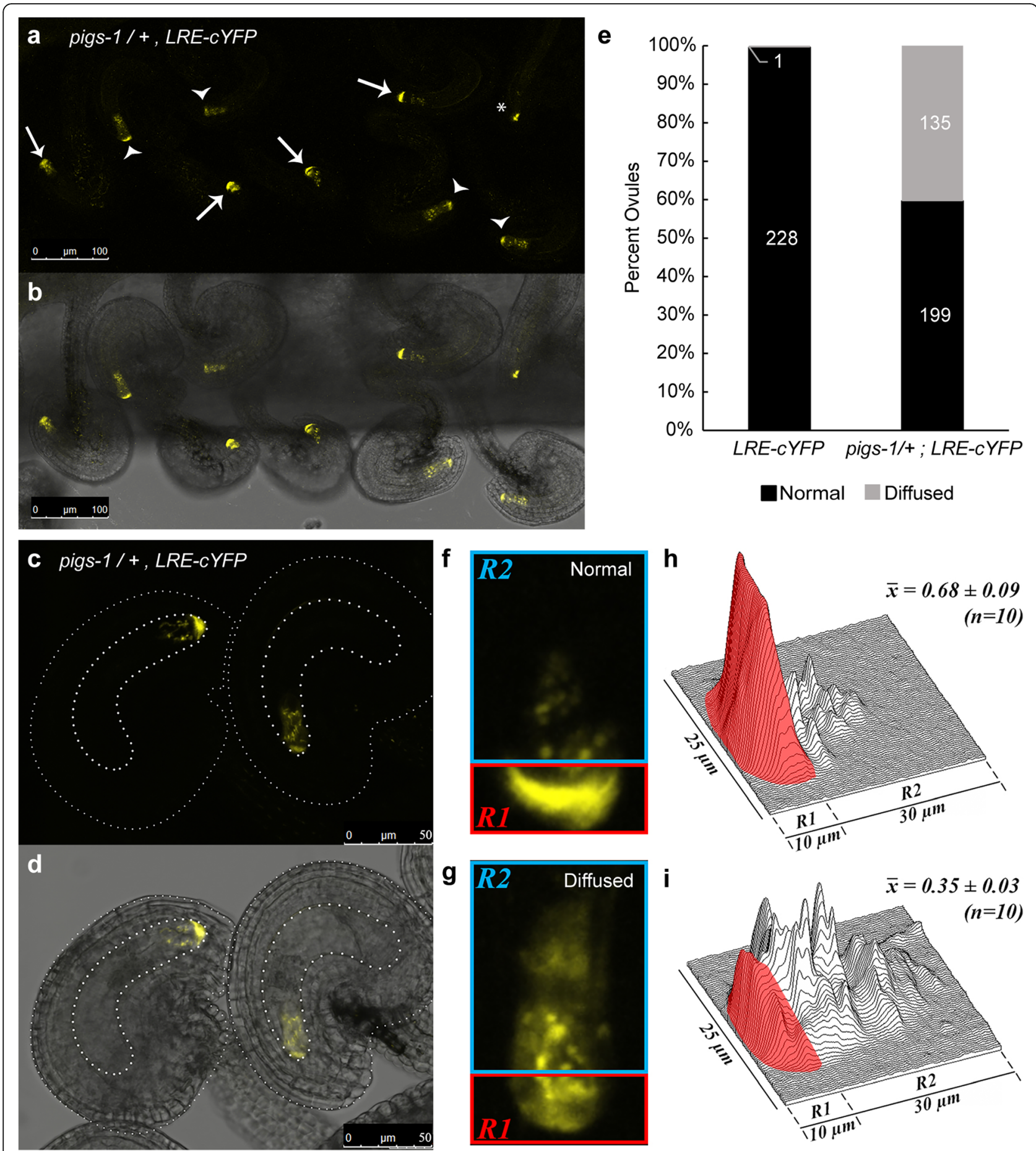

Fig. 1 (See legend on next page.) 
(See figure on previous page.)

Fig. 1 pigs-1 mutation disrupts polar localization of LRE-cYFP in the filiform apparatus of synergid cells. $\mathbf{a}-\mathbf{b}$ Localization of the LRE-cYFP fusion protein in a pigs-1/+ pistil. a Fluorescent image of a portion of the pistil captured in the YFP channel of a confocal microscope and $\mathbf{b}$ Merged view of fluorescent image in (a) with the bright field image of the same portion of the pistil. White arrows, FGs with a polarized cYFP localization in the filiform apparatus; white arrowheads, sibling FGs with a diffuse cYFP localization throughout the synergids; Asterisk, FG not scored for localization due to obscured view from mispostioning of the ovule during mounting on a slide. Bar $=100 \mu$ m. $\mathbf{c}-\mathbf{d}$ Enlarged view of two ovules within a pigs-1/+ pistil Bar $=50 \mathrm{\mu m}$. c Fluorescent image captured in the YFP channel of a confocal microscope and $\mathbf{d}$ Merged view of fluorescent image in (c) with the bright field image of the same ovules captured in (c). e Percent ovules showing normal and diffuse LRE-cYFP localization in synergid cells of wild-type and pigs-1/+ pistils. Numbers in the column refer to total number of ovules scored for indicated categories. $\mathbf{f}-\mathbf{g}$ Representative images of ( $\mathbf{f}$ ) normal and ( $\mathbf{g})$ diffuse LRE-cYFP localization patterns scored within synergid cells of a pigs-1/+ pistil. $\mathbf{h}$-i Surface plots of (h) normal and $\mathbf{i}$ diffuse LRE-cYFP fluorrescent signal scored within synergid cells of a pigs-1/+ pistil. $\bar{X}$ values are average raw integrated densities of CYFP signal in filiform apparatus portion of the synergid cell boxed in red (R1) divided by total signal in R1 + R2, where R2 represents remainder of the synergid cell boxed in blue

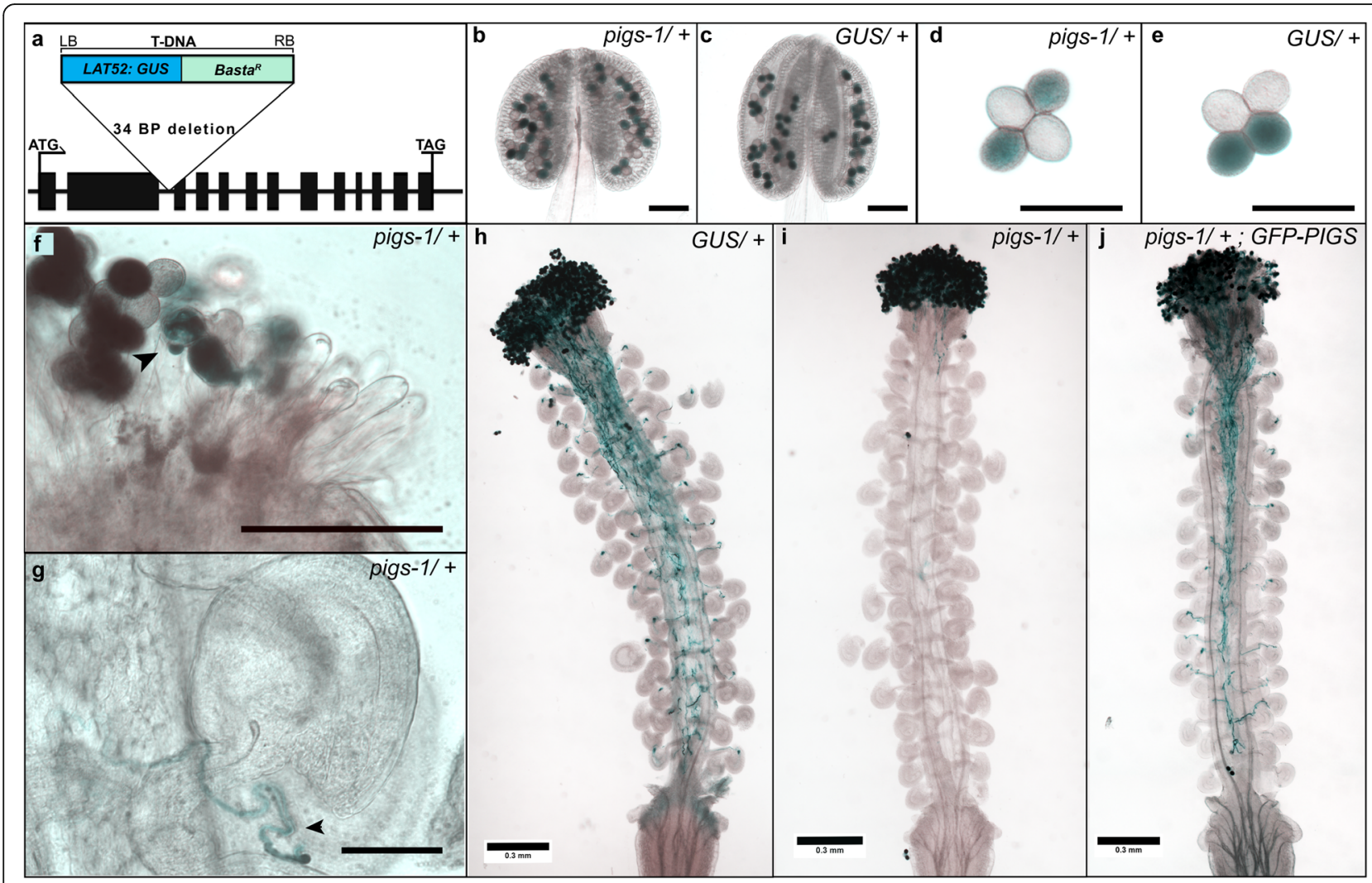

Fig. 2 pigs-1 mutation disrupts pollen tube emergence and growth in vivo. a Gene Model of AtPIG-S (AT3G07180) with a T-DNA insertion (SAIL_162_D06) carrying the basta resistance and pLAT52:GUS genes. Black rectangles indicate exons and lines indicate introns and UTRs. Basta resistance gene is under the control of the 355 promoter and a post meiotic, pollen-specific promoter of LAT52 drives the beta-glucronidase reporter (GUS) gene. Both junctions of T-DNA insertion sites in the AtPIGS gene were sequenced to identify the insertion point in the second intron, which caused a 34 bp deletion. b-c Anther locules containing indehiscent pollen tetrads in pigs-1/+ (b) or wild-type backgrounds (c) were stained with X-Gluc for GUS activity. Scale bar 100 uM. d-e Released pollen tetrads from (d) pigs-1/+ or (e) wild-type anthers show indistinguishable morphology and GUS expression ( $n=100$, in each genotype). Scale bar 50 uM. f Stigma of wild-type pistil pollinated with pigs1/+ stained for GUS activity $18 \mathrm{~h}$ after pollination (HAP). Black arrowhead, pigs-1 pollen showing pollen tube emergence defect. Scale bar $100 \mathrm{uM}$. g Rare instance of pigs-1 pollen tube approaching wild-type ovule within a wild-type pistil pollinated with pigs-1/+ and stained for GUS activity 18 HAP. pigs-1 pollen tube grew along the funiculus and failed to target the micropyle. Black arrowhead, pigs-1 pollen tube growing away from the micropyle. Scale bar 100 UM. h-j Light micrograph of wild-type pistil pollinated with either (h) pLAT52:GUS/+, (i) pigs-1/+, or (j) pigs-1/+ transformed with pPIGS:GFP-PIGS line \#15-3 pollen and stained for GUS activity 18 HAP. Scale bar 300 uM 
Table 2 Limited pollination does not restore male transmission in pigs-1 or gpi8-2

\begin{tabular}{|c|c|c|c|c|c|c|}
\hline \multirow[b]{2}{*}{ Female parent } & \multirow[b]{2}{*}{ Male parent } & \multicolumn{2}{|c|}{ Observed No. of progeny } & \multirow[b]{2}{*}{ TE (R/S) } & \multirow[b]{2}{*}{$x^{2}$} & \multirow[b]{2}{*}{$P$-value } \\
\hline & & Basta $^{\text {Ra }}$ & Basta $^{\mathrm{Sa}}$ & & & \\
\hline Wild type & pigs-1/+ & 3 & 121 & 0.02 & 112.3 & $<0.001$ \\
\hline Wild type & gpi8-2/+ & 1 & 150 & 0.01 & 147.0 & $<0.001$ \\
\hline pigs-1/+ & Wild type & 51 & 71 & 0.72 & 3.28 & 0.07 \\
\hline gpi8-2/+ & Wild type & 47 & 63 & 0.75 & 2.33 & 0.13 \\
\hline
\end{tabular}

$\mathrm{TE}$, Transmission efficiency was calculated as the quotient of basta resistant (R) divided by susceptible (S) progeny of the indicated cross in which only a limited number of pollen grains $(<40)$ were used

X2 was calculated based on the expectation of a 1:1 segregation of basta resistance to susceptibility in the progeny

${ }^{a}$ Basta herbicide resistant $\left(\right.$ Basta $^{\mathrm{R}}$ ) or susceptible (Basta ${ }^{\mathrm{s}}$ ) progeny. Basta resistance gene is linked with the T-DNA that is inserted into the GPI8 or PIGS genes (pigs1 and gpi8-2 mutants, respectively)

benthamiana leaves and checked if the leaves showed transient expression of PIGS-GFP. We detected GFP signal in the $N$. benthamiana leaves that were infiltrated with pPIGS:GFP-PIGS (Additional file 4c and e) but not the leaves that were infiltrated with the helper plasmid only (Additional file $4 \mathrm{~b}$ and d). Interestingly, GFP signal was not detectable 3 days post infiltration, but substantial GFP signal accumulated by 12 days post infiltration suggesting that while the AtPIG-S-GFP fusion protein is stable, the promoter activity of $p P I G S$ used in the construct or incorporation rate of the fusion protein into the GPI-T complex is quite low. Additionaly, consistent with the known residency of the GPI-T complex in the ER, the PIGS-GFP signal in the pavement cells showed a localization pattern that is suggestive of proteins accumulating in the ER (Additional file 4c).

To examine if the pPIGS:GFP-PIGS construct could complement pollen tube emergence and growth defects in pigs-1, we analyzed three independent $\mathrm{T} 1$ transformant lines in a pigs-1/+ background (Lines 7, 15, and 25). In each independent transformant line, we identified $\mathrm{T} 2$ individuals that were heterozygous for the pigs- $1 \mathrm{mu}-$ tation (identified by GUS staining of the tetrads) and carried at least one copy of the pPIGS:GFP-PIGS transgene (based on hygromycin resistance linked to the transgene) and used them in complementation experiments. Wild-type pistils were pollinated with pollen from these T2 plants and stained for GUS activity $18 \mathrm{~h}$ after pollination. In all three independent transformant lines, PIGS-GFP rescued the pollen tube emergence and growth defects in pigs-1 pollen, as pigs-1 pollen tubes (GUS-positive) grew the entire length of the pistils (Fig. 2j). Importantly, the longest pollen tubes in wild-type pistils crossed to pollen from three independent transformants was restored to the full length of the pistil and averaged $2.2 \pm 0.16 \mathrm{~mm}, 2.2 \pm 0.11$, and $2.14 \mathrm{~mm} \pm 0.14$ $\mathrm{mm}$ in length, respectively. Furthermore, presence of the pPIGS:GFP-PIGS transgene rescued the reduced TE of the pigs-1 mutation through the MG, as the TE of the pigs-1 mutation significantly increased from 0.02 without the $p$ PIGS:GFP-PIGS transgene to a TE of 0.76 with the
pPIGS:GFP-PIGS transgene (Table 1). Although pPIGS: GFP-PIGS did not generate a detectable GFP signal in the pollen grains or pollen tubes of any of the three independent transformant lines, the successful complementation of pollen tube growth defects indicates that the transgene is likely expressed. Together, these results indicated that the pollen tube emergence and growth defects in pigs-1 pollen carrying the pPIGS:GFP-PIGS transgene were restored to normal levels.

\section{pigs-1/pigs-1, pPIGS:GFP-PIGS seedlings died after emerging from the seed coat}

Despite this restoration of pollen tube growth, out of 75 $\mathrm{T} 2$ plants from the three independent pPIGS:GFP-PIGS transformant lines, we never detected a T2 plant that produced pollen tetrads homozygous for GUS (4:0 GUS+: GUS- tetrad), the marker linked with the pigs-1 mutation. These results suggested that homozygous pigs-1 plants are not present or occur at a very low frequency among T2 plants due to embryo or seedling lethality. Consequently, we analyzed these transformants to gain insights into the failure to establish pigs-1/pigs-1, pPIGS:GFP-PIGS plants among the progeny of pPIGS: GFP-PIGS complementation lines.

Aborted seeds caused by embryo lethality is one reason for distorted segregation of certain genotypes in progeny and appear as wrinkled brown seeds, as reported in pnt1 mutant that is defective in GPI biosynthesis [12]. No shriveled or wrinkled seeds were observed in any of the three independent complementing pigs-1/+, pPIGS: GFP-PIGS lines. Additionally, immature siliques from line \#15 contained similar number of unfertilized, aborted, and normally developed seeds as in wild type (Additional file 5), suggesting that absence of viable pigs1/pigs-1, pPIGS:GFP-PIGS plants is not due to embryo lethality.

We next performed two experiments to test the possibility that pigs-1/pigs-1, pPIGS:GFP-PIGS plants died at the seedling stage after apparently normal looking seeds germinated. First, selfed seeds from pigs-1/+, pPIGS: GFP-PIGS plants were grown without selection on 
regular MS plates (containing no antibiotic or basta to select for the complementing pPIGS:GFP-PIGS transgene or the pigs-1 mutation, respectively). We found several seedlings were stunted in growth and died shortly after emerging from the seed coat (\% lethality ranging from 18 to 29; Table 3). Presence of lethal seedlings was dependent on the presence of pPIGS:GFP-PIGS transgene, as there were no lethal seedlings in the progeny of pigs-1/+, which lacked the complementing transgene (Table 3). Second, when selfed seeds from pigs-1/+, pPIGS:GFP-PIGS plants, but not from pigs-1/+ plants, were plated on MS plates containing basta (marker to select pigs-1 mutation), besides basta-resistant and basta-susceptible seedlings, we also detected lethal seedlings. Unlike basta-susceptible seedlings, the lethal seedlings contained neither a radicle nor roots and died immediately following emergence from the seed coat (Fig. 3a-c and Table 4). Importantly, lethal seedlings observed on MS basta plates were similar to those observed on regular MS plates, as they did not produce true leaves, failed to develop roots, and embryonic leaves appeared pale in color (Fig. 3c). These results suggested that pigs-1/pigs-1 seeds were established in the presence of pPIGS:GFP-PIGS transgene but died after emerging from the seed coat.

We devised a PCR-based genotyping assay to verify if the lethal seedlings' genotype was indeed pigs-1/pigs-1, pPIGS:GFP-PIGS (Fig. 3d-h and Additional file 6). For this, we scored the three types of seedlings in the progeny of two independent pPIGS:GFP-PIGS transformants grown on MS plates with basta, pooled each type of seedlings, isolated genomic DNA from them, and performed two sets of PCR reactions, one to score presence of the pigs-1 mutation and the other for presence of the pPIGS:GFP-PIGS transgene and wild-type copy of AtPIG-S (Fig. 3d-h and Additional file 6). We found that only the pooled lethal seedlings, but not basta-resistant or basta-susceptible seedlings, were homozygous for the pigs-1 mutation, confirming the genotype of lethal seedlings is indeed pigs-1/pigs-1, pPIGS:GFP-PIGS (Fig. 3g and $\mathrm{h}$ and Additional file 6). Care was taken to prevent

Table 3 Seedling lethality of pigs-1/pigs-1; pPIGS:GFP-PIGS on MS plates

\begin{tabular}{|c|c|c|c|}
\hline \multirow[t]{2}{*}{ Selfed parent } & \multicolumn{2}{|c|}{ Observed No. of progeny } & \multirow{2}{*}{$\begin{array}{l}\% \\
\text { Lethal }\end{array}$} \\
\hline & Normal & Lethal & \\
\hline pigs-1/+ & 346 & 0 & 0 \\
\hline pigs-1/+; GFP-PIGS \#15-3 & 315 & 78 & 19.85 \\
\hline pigs-1/+; GFP-PIGS \#7-25 & 140 & 32 & 18.60 \\
\hline pigs-1/+; GFP-PIGS \#25-6 & 324 & 127 & 28.16 \\
\hline
\end{tabular}

Selfed seeds were placed onto MS plates containing no antibiotic or basta Seedling phenotypes were scored according to images and descriptions in Fig. 3

GFP-PIGS refers to three independent transformant T2 lines of pPIGS:GFP-PIGS seed coat contamination during seedling collection so that the maternal diploid genotype of the seed coat (pigs-1/+,GFP-PIGS) did not confound the PCR-based genotyping of seedlngs. Indeed, lack of endogenous wild-type AtPIG-S band in the lethal seedling samples confirmed that our seedling collection of this sample was free of seed coat contamination (Fig. 3h and Additional file 6). Additionally, the PCR results showed that the pPIGS:GFP-PIGS transgene was segregating in both pooled basta-resistant and basta-susceptible seedlings, and that pooled basta-susceptible seedlings did not contain the pigs- 1 mutation as expected (Fig. $3 g$ and $\mathrm{h}$ and Additional file 6). These results showed that although the pPIGS:GFP-PIGS construct could rescue the pollen tube growth defects of pigs-1 mutant pollen and allowed homozygous pigs-1/pigs-1 seeds to be formed, the AtPIGS-GFP expressed from native PIGS promoter couldn't complement the developmental defects in pigs1/pigs-1 seedlings. Alternatively, it is possible that an independent mutation in another gene that is tightly linked to the pigs-1 T-DNA caused the seedling lethality of pigs-1/pigs-1 pPIGS:GFP-PIGS seedlings.

\section{Discussion \\ Deficiency in GPI-anchoring of GAPs does not appear to disrupt the FG functions}

Like that seen in cis mutations in the GAS of LRE (a substrate of GPI-T) and in gpi8-2 (a component of GPI$\mathrm{T})$, pigs-1 also disrupts the polar LRE-cYFP localization in the FA without affecting its own transmission through the FG. The GPI anchor of ZERZAUST, another Arabidopsis GAP, is dispensable for its biological activity [40], so it's possible that LRE could simply be another GAP whose GPI anchor doesn't influence its function. If so, the GAS in LRE could simply be a relic of its duplication where the ancestral copy contained a more functionally relevant GAS.

Still, presumably in pigs-1 FGs, all GAPs lack GPI anchors, so it is unlikely that GPI anchor addition is unimportant for the function of all FG-expressed GAPs. One possibility is that retention of mature GAPs from the diploid pigs-1/+ megaspore mother cell (MMC), after meiosis and the three mitotic cell divisions in megagametogenesis, could support the function of the mature pigs-1 FG. In support of this hypothesis, sporulating diploid yeast cells with a disruption in the GPI2 gene produce GPI-deficient haploid ascospores that are able to germinate and complete up to four mitotic cell divisions [23]. Another possibility is that mature GAPs could be supplied by integument tissues that surround the FG, as it is known 'membrane painting' allows transfer of GAPs between cell surfaces of animal cells upon contact [27, 28]. Consistent with either of these 


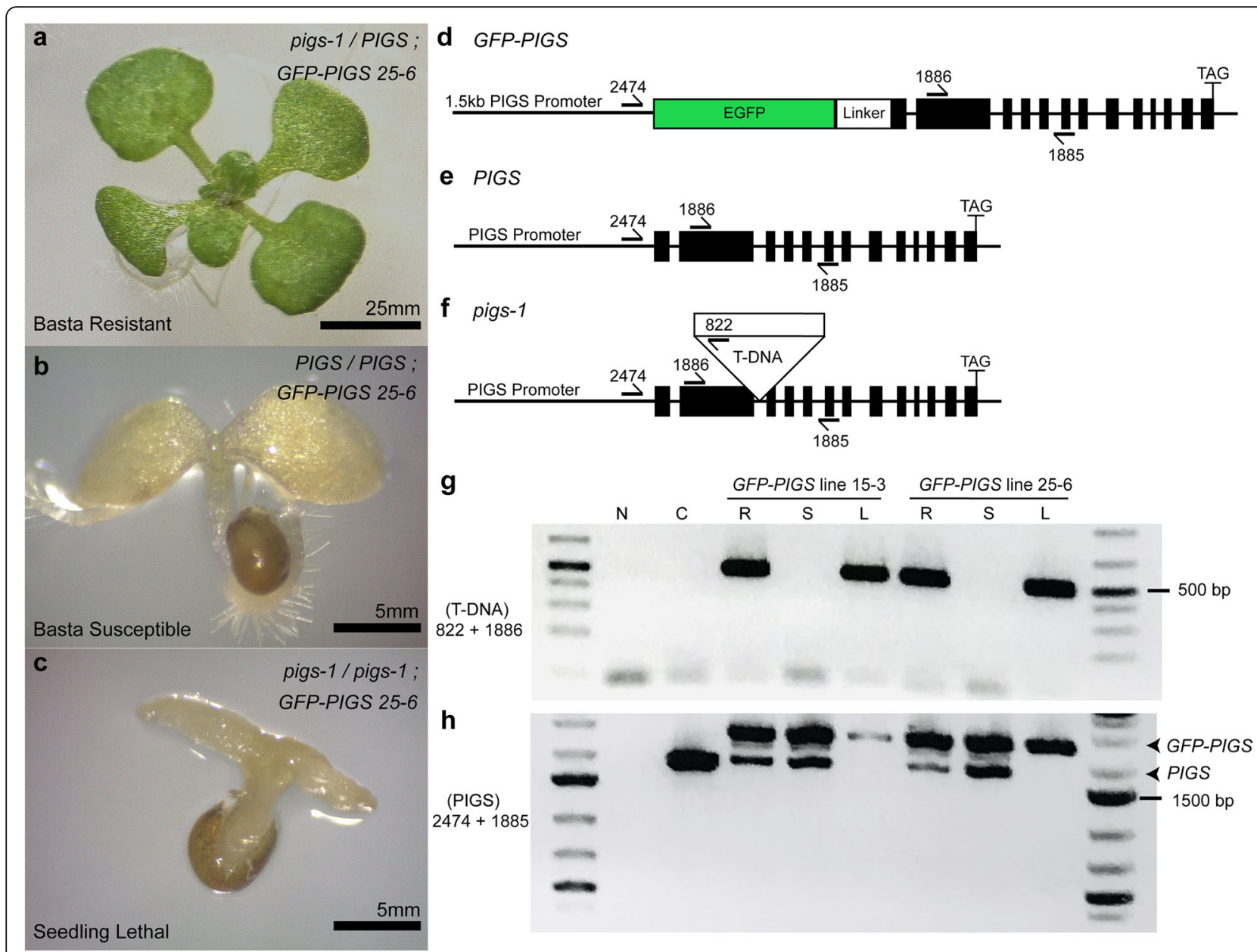

Fig. 3 Lethal seedlings segregated in the progeny of selfed pigs-1/t, pPIGS:GFP-PIGS. a-c Light micrographs of seedlings from selfed seeds of pigs1/+, pPIGS:GFP-PIGS line \#25-6 grown on MS plates supplemented with basta for 14 days in constant light. a Basta-resistant pigs-1/+, pPIGS:GFPPIGS seedlings had well developed true green leaves and prolific root, root hairs, and lateral root growth. $\mathbf{b}$ Basta-susceptible $+/+$, pPIGS:GFP-PIGS seedlings had minimal to no true leaves or root hair and lateral root development. c Lethal pigs-1/pigs-1, pPIGS-PIGS-GFP seedlings were small, showed aberrant pale cotyledon growth, and lacked radicle or roots. *Images shown were taken using a Zeiss Axiovert 100 microscope with either an 8x objective, Bar $=5 \mathrm{~mm}$ (a) or 50x objective, Bar $=5 \mathrm{~mm}$ (b-c). $\mathbf{d}-\mathbf{f}$ Gene model of the (d) pPIGS:GFP-PIGS construct, (e) endogenous AtPIG-S gene, and (f) pigs-1 T-DNA insertion. Primers used in the PCR-based genotyping of each of these three DNAs are indicated. $\mathbf{g}$-h PCR-based genotyping using gDNA isolated from basta-resistant, basta-susceptible, and lethal seedling progeny of PIGS-GFP T2 lines \#15-3 and \#26-6 for either the presence of the pigs-1 T-DNA using primers $822+1886 \mathrm{~g}$ ) or for the presence of the endogenous AtPIG-S or GFP-PIGS gene using primers $2474+1885$ (h). Expected size for endogenous AtPIG-S is $1896 \mathrm{bp}$ and expected size for GFP-PIGS is 2586 bp. Original, un-cropped gels are provided in Additional file 6. N, water control; C, Columbia-0 gDNA; R, basta-resistant; S, basta susceptible; L, lethal seedlings. *All primer sequences listed in Additional Table 1

possibilities, several GAPs involved in FG development such as AGP4, AGP18, A36, and A39 have mutant defects that are sporophytic in nature [1, 10,33].

Still, GAPs involved in pollen tube reception such as LRE and ENODLs are gametophytic in nature [17] and so loading from sporophytic MMC or integument tissues cannot completely explain why GAP-deficient FGs could still function normally. Interestingly, both LRE and ENODLs function in synergid cells, a polarized cell whose default secretory pathway is to direct protein traffic to the FA [35]. The high density of cell wall and membrane invaginations of the FA combined with the hydrophobicity of the un-cleaved GAS region of GAPs in pigs-1 synergids could facilitate their sequestration in the FA [8]. The synergid-secreted GAPs, ENODLs and LRE, could then be further retain at the pigs-1 FA by virtue of their binding to ectodomains of receptor like kinases, such as FER $[24,25]$.

Finally, while gpi8-2 and pigs-1 mutations disrupt the polar localization of LRE-cYFP in the FA, some LREcYFP molecules still reach the FA of pigs-1 (Figs. 2a-g) and gpi8-2 [25] synergids. GAPs have the capacity to function at very low concentrations due to the high membrane dynamics of GPI anchors and their ability to 
Table 4 Transmission of pigs-1 mutation in self-pollinated pistils

\begin{tabular}{|c|c|c|c|c|c|}
\hline \multirow[t]{2}{*}{ Selfed parent } & \multicolumn{3}{|c|}{ Observed No. of progeny } & \multirow[b]{2}{*}{$x^{2}$} & \multirow[b]{2}{*}{$P$-value } \\
\hline & Basta $^{R}$ & Basta $^{S}$ & Lethal & & \\
\hline$+/+;$ BastaR/t+ & 130 & 43 & 0 & 0.001 & 0.97 \\
\hline pigs-1/+ & 151 & 153 & 0 & 52.01 & $<0.001$ \\
\hline pigs-1/+; GFP-PIGS \#15-3 & 512 & 256 & 166 & 101.4 & $<0.001$ \\
\hline pigs-1/+; GFP-PIGS \#7-25 & 152 & 74 & 65 & 40.22 & $<0.001$ \\
\hline pigs-1/+; GFP-PIGS \#25-6 & 402 & 154 & 112 & 44.61 & $<0.001$ \\
\hline
\end{tabular}

Basta herbicide-resistant $\left(\right.$ Basta $\left.^{\mathrm{R}}\right)$ or susceptible (Basta ${ }^{\mathrm{S}}$ ) progeny. Basta resistance is linked with the T-DNA that is inserted into either the AtPIGS gene (pigs-1 mutant) (Fig. 2a) or in an unknown location in the genome of the +/+; BastaR/+ (positive control line) [21]

X2 was calculated based on the expectation of a 3:1 segregation of basta resistance to susceptibility in the progeny

Seedling phenotypes scored according to images and descriptions in Fig. 3 GFP-PIGS refers to three independent transformant T2 lines of pPIGS:GFP-PIGS

rapidly "patrol" membranes $[29,38]$. Therefore, a $50 \%$ decrease in LRE-cYFP localization to the FA may not be sufficient to negatively impact its ability to function. In summary, the unusual structural and functional properties of synergids may overcome GPI anchor addition deficiencies to synergid cell-expressed GAPs and confound genetic analysis of GAPs in synergid cells.

\section{Loss of AtPIG-S function led to defects in pollen tube emergence and tube growth}

Contrary to pigs-1 mutant FGs, pigs-1 mutant MGs have severe fertility defects. Here, by utilizing the GUS reporter within the pigs-1 T-DNA we show for the first time that loss of AtPIG-S function in pollen (and by inference GPI transamidation-deficient pollen) have defects at the stages of pollen tube emergence and growth. Future investigations could focus on testing if low TE reported previously in gpi8-1 and gpi8-2 MGs are also caused by defects in pollen tube emergence and tube growth. It's possible that loss of GPI anchor addition to pollen-expressed GAPs such as LLG2 and LLG3 (paralogs of LRE), which are critical for maintaining the cell wall integrity of pollen tubes, could be less tolerated in pollen than the FG because of the rapid developmental process pollen must undergo during tube emergence and growth [11]. While experiments on localization of LLG2/3 in pigs- 1 pollen tubes would be difficult due to the low level of pigs-1 pollen tube growth, analyzing the effect of cis mutations in the GAS region of LLG2/3, similar to those reported in LRE [25], could substantiate our findings on the increased importance of GPI anchor addition to GAPs in the MG compared to that in the FG.

Our results on pigs-1 (this study) and gpi8-2 [25] causing severe MG defects are also consistent with previous reports on mutations in four GPI biosynthesis genes causing a decrease in male fertility [4, 12, 22]. While the mutant analyses on all four of these GPI biosynthesis genes established an important role of GPI in MG function, they could not preclude the possibility that the absence of free, non-protein linked GPI underlies these MG defects. In addition to serving as a substrate in the transamidation reaction catalyzed by GPI-T, evidence in protists suggests that free GPI plays an independent and more essential role in cell expansion than mature GAPs $[15,19]$. Here, by targeting the downstream transamidation step rather than GPI anchor biosynthesis, our study provides support to the possibility that the pollen tube emergence and growth defects in pigs-1 pollen grains are due to a lack of GPI anchor attachment to GAPs. Still, free GPI may be important in pollen, but to our knowledge plasma membranelocalized free GPI in pollen has not been documented. Because pigs-1 pollen grains are viable until tube emergence, they also offer a potential in vivo system to study free GPI in plants, as free GPI likely accumulates in pigs1 pollen grains, similar to GPI transamidation-deficient mutants in yeast [30].

\section{A possible role for AtPIG-S in Arabidopsis early stage seedlings is revealed by rescue of AtPIG-S function in the male gametophyte}

Successful restoration of the pigs-1 pollen tube growth defects by the pPIGS:GFP-PIGS transgene confirmed the role of AtPIG-S in the MG and suggested that fusion of GFP to the AtPIG-S protein did not affect its function in the GPI-T complex. Hence, GFP-PIGS fusion protein could be used to immunoprecipitate and unravel the subunit composition of the GPI-T complex in Arabidopsis similar to HA-PIG-S, which was used to pull down the GPI-T complex in CHO cells [16]. However, it should be noted that in yeast, the PIG-S ortholog, GPI17, neither co-purifies [7] nor stably interacts with other subunits of GPI-T [43].

Rescue of AtPIG-S function in the MG by the pPIGS: GFP-PIGS transgene also allowed us to establish pigs-1/ pigs-1 seedlings. Like seedlings with a mutation in $P E A$ NUT1 (PNT1), a predicted mannosyltransferase required for GPI biosynthesis [12], we showed that pigs-1/pigs-1 seedlings carrying the pPIGS:GFP-PIGS transgene emerged from the seed coat but soon became necrotic. However, unlike pnt1 mutant, the pigs-1/pigs-1 seeds carrying the PPIGS:GFP-PIGS transgene were not wrinkled and shriveled. Previously, a role for GPI transamidation in seedling development and later stage plant development was proposed after characterizing the nonlethal allele, gpi8-1, a missense mutation in AtGPI8 [2]. However, the aboveground organs of gpis-1 mutants were very minimally affected even 15 days after germination and produced roots, albeit significantly shorter than wild type. In contrast, pigs-1/pigs-1, pPIGS:GFPPIGS seedlings died directly following emergence from 
the seed coat. Notably, the maternal seed coat is pigs$1 /+$ and perhaps haplo-sufficient to support the development of pigs-1/pigs-1, pPIGS:GFP-PIGS embryos.

Still, it is not clear why the pPIGS:GFP-PIGS transgene failed to complement seedling function, though it was previously reported that in Arabidopsis, a AtGPI8-EGFP construct also driven under 2.1-kb of it's endogenous promoter did not complement all gpi8-1 phenotypes and that fluorescence reporter in this construct was not detectable [2]. It is therefore possible that the promoter sequences used in our construct also lacked the cis elements required for sufficient expression in germinated seedlings, where AtPIG-S is expressed (http://bar.utoronto.ca/eplant/). Our results reported here does not rule out an alternative cause for the seedling lethality detected in the progeny of pigs-1/pigs-1, pPIGS:GFP-PIGS. It is possible that a tighlty linked mutation or a second T-DNA insertion in a neighboring gene could be cosegregating with the pigs-1 T-DNA and resulted in the seedling lethality. Performing complementation experiments with pPIGS:GFP-PIGS in additional alleles of AtPIGS would help test this possibility.

\section{Conclusions}

During flowering plant reproduction, GAPs mediate many MG and FG functions. While the loss of GPI anchor addition to the GAPs did not result in noticeable phenotype(s) in the FG, its importance in the MG is underscored by the pollen tube emergence and growth defects of pigs-1 mutant pollen. Up to this point, MG defects in GPI anchor biosynthesis mutants could not preclude the possibility that the absence of free, nonprotein linked GPI underlie their MG defects. Here, instead we analyzed a putative subunit in the GPI-T complex, which is involved in post-translational addition of GPI anchors to GAPs, and show that MG defects of GPI-deficient pollen grains seen previously are likely due to a lack of mature GAPs.

We also substantiate the finding that loss of GPI anchor addition to the GAPs in the FG does not affect its functions. While it's possible the megaspore mother cell could have supplied mature GAPs to the FG across cell divisions, the pollen tube reception defects seen in the FG-expressed GAPs such as LRE and ENODLs are gametophytic in origin. Therefore, we propose that the importance of their GPI anchors in the FG-expressed GAPs could be masked by their synergid expression such that (1) the specialized secretion system of synergids is sufficient to transport non-GPI anchored GAPs to the plasma membrane, and (2) the high surface area of the FA in combination with the hydrophobicity of their un-cleaved GAS region are sufficient for their sequestration and function at the FA. For these reasons, the MG, compared to FG, is a better haploid model to examine the role of GAPs.

\section{Methods}

\section{Plant materials and growth conditions}

Columbia (Col-0) is the ecotype of all Arabidopsis seeds used in this study. The gpi8-2/+ (CS853564), pigs-1/+ (CS807841), and pigt-1 (Salk_099158) seeds were purchased from Arabidopsis Biological Resource center (ABRC) in Columbus, Ohio. The pLAT52:GUS gene in the wild-type background was described previously [21] and is a donation from Dr. Mark Johnson, Brown University, Providence, RI, USA. Once the manuscript has been published, newly established transgenic lines generated as part of this study - pLRE:LRE-cYFP/pLRE:LREcYFP, pigt-1/pigt-1; pLRE:LRE-cYFP/pLRE:LRE-cYFP, pigs-1/+, and pPIGS:GFP-PIGS, pigs-1/+ - by Nick Desnoyer, the first author of this study, will be deposited in the Arabidopsis Biological Resource Center in Columbus, Ohio, USA. All Arabidopsis plants reported in this study were grown as described [39].

\section{Pollen tube growth assays}

In vivo pollen tube growth assays were done as described [39]. Briefly, pLAT52:GUS or pigs-1/+ pollen was crossed to emasculated stage 14 pistils of the indicated plants. Crossed pistils were then collected $18 \mathrm{~h}$ after pollination, fixed in $80 \%$ acetone, stained for GUS activity using $\mathrm{x}$-gluc [21], mounted in 50\% glycerol, and imaged using differential interference contrast optics in a Zeiss Axiovert 100 microscope. Light micrographs of pistils were used to measure pollen tube length using ImageJ.

\section{Transmission efficiency assay}

Reciprocal crosses were done on emasculated stage 14 pistils. For limited pollination experiments, less than 40 pollen grains were used to pollinate pistils under a dissecting microscope. Seeds from these crosses were placed on plates containing appropriate antibiotics/herbicides corresponding to the antibiotic or herbicide resistance marker in the T-DNA. 14-day old seedlings with true leaves and developing roots were scored as resistant.

\section{Seedling lethal assay}

Seeds were plated on either MS plates with or without basta, stratified for 2 days at $4{ }^{\circ} \mathrm{C}$, incubated in the growth chamber for 14 days and scored under a dissecting scope. In basta plates, three types of seedlings were scored. If seedlings contained true leaves and all leaves were green, and had robust root growth with root hairs and secondary roots, they were scored as 'basta-resistant'. If the germinated seedlings contained fully developed embryonic leaves but true leaves never emerged, 
and radicle and roots emerged but failed to grow and after 2 weeks the seedling died, they were scored as 'basta-susceptible'. Finally, if seedlings that began to emerge from the seed coat contained only embryonic leaves and contained neither radicle nor roots, they were scored as 'lethal seedlings'.

In case of seeds plated on MS plates without basta, two types of seedlings were scored; if seedlings contained true leaves and all leaves were green, and had robust root growth with root hairs and secondary roots, they were scored as 'normal' and if seedlings that began to emerge from the seed coat contained only embryonic leaves and contained neither radicle nor roots, they were scored as 'lethal' seedlings.

For genomic DNA isolation, seedlings were collected after 14 days of growth, taking care not to contaminate the collection with seed coat, especially in case of lethal seedlings. Although lethal seedlings were detected within 7-10 days after plating, we scored and collected the three types of seedlings after 14 days of growth so that it was possible to separate and collect lethal seedlings without seed coat sticking to the seedling and contaminating the collection. Avoiding seed coat contimation was essential so that diploid maternal genotype of the seed coat did not confund the PCR genotyping of seedlings. Each of the three type of seedlings were collected separately, pooled and frozen in liquid nitrogen, and genomic DNA was isolated from them as described previously [39]. PCR was performed with at least $100 \mathrm{ng}$ of genomic DNA as a template in each reaction as follows: Step 1: (1X) $98.0^{\circ} \mathrm{C}$ for $2 \mathrm{~min}$; Step 2: $(34 \mathrm{X}) 95.0{ }^{\circ} \mathrm{C}$ for $30 \mathrm{~s}$, $56.0^{\circ} \mathrm{C}$ for $20 \mathrm{~s}$, and $72.0^{\circ} \mathrm{C}$ for $1 \mathrm{~min}$; Step 3: (1X): $72.0^{\circ} \mathrm{C}$ for $2 \mathrm{~min}$. PCR products were separated on a $1 \%$ agarose gel. Sequence of primers used and expected size of PCR products are listed in Additional Table 1.

\section{Confocal microscopy}

The pigt-1/pigt-1 or pigs-1/+ mutant was crossed to $p L R E$ : LRE-cYFP/pLRE:LRE-cYFP [25] and established pigt-1/ pigt-1, pLRE:LRE-cYFP/pLRE:LRE-cYFP and pigs-1/+, $p L R E: L R E-c Y F P / p L R E: L R E-c Y F P$ lines, respectively. Confocal images of ovules from these lines were taken after mounting unpollinated stage 14 pistils. Fluorescent images were taken using a Leica SP5 confocal laser scanning microscope system. For cYFP imaging, samples were excited with a 488-nm laser, and emission spectra between 510 and $550 \mathrm{~nm}$ were collected. YFP images were processed with ImageJ software (http://imagej.nih.gov/ij/). YFP localization was quantified by measuring raw integrated density using the ROI manager tool in ImageJ.

\section{RNA isolation, RT PCR, and RT-qPCR}

RNA was isolated according to [36]. Briefly, for each biological replicate, 14 days-old pigt-1/pigt-1 and Col-0 seedlings were collected, flash frozen, and stored at $80^{\circ} \mathrm{C}$ until RNA extraction. Three biological replicates were collected for both genotypes. RNA was isolated and quality checked as described in [25]. For RT-PCR, cDNA was synthesized from $2 \mu \mathrm{g}$ total RNA using SuperScript $^{\text {tw }}$ IV First-Strand Synthesis System, ThermoFisher Scientific, Catalog \#18091050.

PIGT PCR on pigt-1/pigt-1 and Col-0 cDNA was amplified using either primer pairs (P1 + P2) or (P1 + P4) with the following program: Step 1 (1X): $98.0^{\circ} \mathrm{C}$ for $2 \mathrm{~min}$; Step 2 (37X): $98.0^{\circ} \mathrm{C}$ for $15 \mathrm{~s}, 55.0^{\circ} \mathrm{C}$ for $15 \mathrm{~s}$, and $72.0^{\circ} \mathrm{C}$ for 2 min. Sequence of primers used and expected size of PCR products are listed in Additional Table 1.

The following qPCR program was used for all qPCR experiments: Step 1 (1X): $95.0^{\circ} \mathrm{C}$ for $10 \mathrm{~min}$; Step 2: (40X) $95.0^{\circ} \mathrm{C}$ for $10 \mathrm{~min}, 55.0^{\circ} \mathrm{C}$ for $30 \mathrm{~s}$ and $72.0^{\circ} \mathrm{C}$ for $30 \mathrm{~s}$, Data collection and real-time analysis enabled; Step 3 (101X): $45.0^{\circ} \mathrm{C}-95.0^{\circ} \mathrm{C}$ for $10 \mathrm{~s}$, increase set point temperature after Step 2 by $0.5^{\circ} \mathrm{C}$, Melt curve data collection and analysis enabled. $\mathrm{Ct}$ values were normalized to $A C T I N 2 / 8$. Relative levels of gene expression were calculated according to [36]. At least two technical replicates of qPCR were performed for each experiment. Primers used in qPCR reactions are listed in Additional Table 1.

\section{Cloning pPIGS:GFP-PIGS}

The $p$ PIGS:GFP-PIGS construct was created by overlap PCR with PrimeSTAR ${ }^{\circ}$ GXL DNA Polymerase (TaKaRa Bio Inc.; Catalog \# R050A) and DNA templates and primers listed in Additional Table 1 and cloned into pH7WG plasmid linearized with SalI-HF (NEB, Cataog \# R3138S) and AscI (NEB, Catalog \# R0558S) by using the In-Fusion HD Cloning Plus system (Clontech, Catalog \# 639645). The recombinant plasmids were transformed into Stellar ${ }^{\mathrm{rm}}$ Competent Cells (Clontech, Catalog \# 636763), and positive colonies were selected on LB plates containing spectinomycin $(100 \mu \mathrm{g} / \mathrm{mL}$, SigmaAldrich, Catalog \# 85555). The construct was sequence verified (Eton Bioscience, Inc.) before transforming into Agrobacterium tumefaciens (GV3101 pMP90 strain). The positive colony selected for transforming into Arabidopsis was also verified by colony PCR for the presence of the transgene.

\section{Plant transformation}

pigs-1/+ heterozygous inflorescences were dipped into transformation solution containing Agrobacterium tumefaciens (GV3101 pMP90 strain) harboring the pPIGS: GFP-PIGS plasmid [3]. Hygromycin-resistant transformants were selected as described in [25] based on the protocol originally reported in [13] with a Hygromycin concentration of $20 \mu \mathrm{g} / \mathrm{mL}$. Transformants were selected based on presence of true leaves, which were present only in hygromycin-resistant plants. 


\section{Transient expression of pPIGS:GFP-PIGS in Nicotiana benthamiana}

An Agrobacterium GV3101::pMP90 strain carrying the plasmid containing $p$ PIGS:GFP-PIGS was used to transiently express GFP-PIGS fusion protein in $N$. benthamiana as described [42]. A leaf punch was taken 3 days or 12 days post-infiltration from the infiltrated area and mounted onto $50 \%$ glycerol. The bottom epidermis of the leaf disk was then analyzed by confocal microscopy. Images were taken using a $488 \mathrm{~nm}$ wavelength at $10.5 \%$ laser power on a Zeiss LSM 880 AxioObserver.

\section{Image processing}

Photoshop CC 2018, Illustrator CC 2019 (Adobe, https://www.adobe.com/) and ImageJ were used for assembling image panels and preparing figures.

\section{Accession numbers}

Accession numbers of genes studied in this work: LRE (At4g26466), AtGPI8 (At1G08750), and AtPIGT (At3G07140).

\section{Supplementary information}

Supplementary information accompanies this paper at https://doi.org/10. 1186/s12870-020-02587-x.

Additional file 1: pigt-1 mutation does not affect polar localization of LRE-CYFP in the filiform apparatus of synergid cells.

Additional file 2: Analysis of PIG-T expression in the pigt-1 mutant.

Additional file 3: Pollen germination and tube emergence defects.

Additional file 4: Transient expression of AtGFP-PIGSprotein in Nicotiana benthamiana leaves.

Additional file 5: Selfed seed set in T2 pigs-1/+, pPIGS:GFP-PIGS plants. Additional file 6: PCR-based genotyping of pigs-1/pigs-1, pPIGS:GFP-PIGS plants

Additional file 7: Table S1. List of primers used in this study.

\section{Abbreviations}

GPI: Glycosylphosphatidylinositol; GPI-T: GPI transamidase complex GAPs: GPI-anchored proteins; FG: Female Gametophyte; MG: Male Gametophyte; LRE: LORELEl; FA: Filiform Apparatus; FER: FERONIA; TE: Transmission Efficiency; MMC: Megaspore Mother Cell

\section{Acknowledgements}

This research was supported by a NSF grant to R.P. (IOS-1146090) and the Boynton Graduate Fellowship in Plant Molecular Biology, School of Plant Sciences, University of Arizona and University of Arizona Graduate Professional Student Council to N.D. We thank Dr. Xunliang Liu for generating the pigt/pigt, $P L R E: L R E-C Y F P$ line. We thank the Yadegari lab for help with transient expression of pPIGS:GFP-PIGS in Nicotiana benthamiana. We thank Patty Jansma in the Marley Imaging Core Facility for help with confocal microscopy.

\section{Authors' contributions}

N.D. and R.P. designed the experiments; N.D. established all three transgenic lines ( $p$ LRE:LRE-CYFP/pLRE:LRE-CYFP, pigt-1/pigt-1; LRE:LRE-CYFP/pLRE:LRE-CYFP, pigs-1/+, and pPIGS:GFP-PIGS, pigs-1/+) generated newly as part of this study. N.D., G.H., and E.J. performed the experiments; N.D. and R.P. wrote the paper. All authors have read and approved the manuscript.

\section{Funding}

This research was supported by a NSF grant to R.P. (IOS-1146090). N.D. was supported by the Boynton Graduate Fellowship in Plant Molecular Biology, School of Plant Sciences, University of Arizona and University of Arizona Graduate Professional Student Council. None of these funding bodies were involved in designing the study, collecting, analysing or interpreting data, or in writing the manuscript.

\section{Availability of data and materials}

The datasets used and/or analysed during the current study are available from the corresponding author on reasonable request.

\section{Ethics approval and consent to participate}

Not applicable.

\section{Consent for publication}

Not applicable.

\section{Competing interests}

The authors declare that they have no competing interests. The corresponding author is an associate editor of BMC Plant Biology. The corresponding author had no role in the editorial process.

\section{Author details}

${ }^{1}$ School of Plant Sciences, University of Arizona, Tucson, AZ 85721, USA.

${ }^{2}$ Present Address: Department of Plant and Microbial Biology, University of Zurich, Zollikerstrasse 107, CH-8008 Zurich, Switzerland.

Received: 27 April 2020 Accepted: 3 August 2020

Published online: 18 August 2020

\section{References}

1. Acosta-Garcia G, Vielle-Calzada JP. A classical arabinogalactan protein is essential for the initiation of female gametogenesis in Arabidopsis. Plant Cell. 2004:16:2614-28.

2. Bundy MG, Kosentka PZ, Willet AH, Zhang L, Miller E, Shpak ED. A mutation in the catalytic subunit of the Glycosylphosphatidylinositol Transamidase disrupts growth, fertility, and stomata formation. Plant Physiol. 2016;171: 974-85.

3. Chung MH, Chen MK, Pan SM. Floral spray transformation can efficiently generate Arabidopsis transgenic plants. Transgenic Res. 2000;9:471-6.

4. Dai XR, Gao XQ, Chen GH, Tang LL, Wang H, Zhang XS. Abnormal pollen tube guidance1, an endoplasmic reticulum-localized Mannosyltransferase homolog of Glycosylphosphatidylinositol10 in yeast and

Phosphatidylinositol Glycan Anchor Biosynthesis B in human, is required for Arabidopsis pollen tube Micropylar guidance and embryo development Plant Physiol. 2014;165:1544-56.

5. Duan $\mathrm{QH}$, Kita D, Johnson EA, Aggarwal M, Gates L, Wu HM, Cheung AY. Reactive oxygen species mediate pollen tube rupture to release sperm for fertilization in Arabidopsis. Nat Commun. 2014;5:3129-229.

6. Ellis M, Egelund J, Schultz CJ, Bacic A. Arabinogalactan-proteins: key regulators at the cell surface? Plant Physiol. 2010;153:403-19.

7. Fraering P, Imhof I, Meyer U, Strub JM, Van Dorsselaer A, Vionnet C, Conzelmann A. The GPI transamidase complex of Saccharomyces cerevisiae contains Gaa1p, Gpi8p, and Gpi16p. Mol Biol Cell. 2001;12:3295-306.

8. Galian C, Bjorkholm P, Bulleid N, Von Heijne G. Efficient Glycosylphosphatidylinositol (GPI) modification of membrane proteins requires a C-terminal anchoring signal of marginal hydrophobicity. J Biol Chem. 2012:287:16399-409.

9. Gamage DG, Hendrickson TL. GPI transamidase and GPI anchored proteins: oncogenes and biomarkers for cancer. Crit Rev Biochem Mol Biol. 2013;48: 446-64.

10. Gao H, Zhang YH, Wang WL, Zhao KK, Liu CM, Bai L, Li R, Guo Y. Two membrane-anchored aspartic proteases contribute to pollen and ovule development. Plant Physiol. 2017;173:219-39.

11. Ge Z, Zhao Y, Liu MC, Zhou LZ, Wang L, Zhong S, Hou S, Jiang J, Liu T, Huang Q, Xiao J, Gu H, Wu HM, Dong J, Dresselhaus T, Cheung AY, Qu LJ. LLG2/3 are co-receptors in BUPS/ANX-RALF signaling to regulate Arabidopsis pollen tube integrity. Curr Biol. 2019;29(19):3256-65 e5.

12. Gillmor CS, Lukowitz W, Brininstool G, Sedbrook JC, Hamann T, Poindexter P, Somerville C. Glycosylphosphatidylinositol-anchored proteins are required 
for cell wall synthesis and morphogenesis in Arabidopsis. Plant Cell. 2005;17: 1128-40.

13. Harrison SJ, Mott EK, Parsley K, Aspinall S, Gray JC, Cottage A. A rapid and robust method of identifying transformed Arabidopsis thaliana seedlings following floral dip transformation. Plant Methods. 2006;2:19.

14. Higashiyama $\mathrm{T}$, Takeuchi $\mathrm{H}$. The mechanism and key molecules involved in pollen tube guidance. Annu Rev Plant Biol. 2015;66(66):393-413.

15. Hilley JD, Zawadzki UL, Mcconville MJ, Coombs GH, Mottram JC. Leishmania mexicana mutants lacking glycosylphosphatidylinositol (GPI): protein transamidase provide insights into the biosynthesis and functions of GPIanchored proteins. Mol Biol Cell. 2000;11:1183-95.

16. Hong YJ, Ohishi K, Kang JY, Tanaka S, Inoue N, Nishimura J, Maeda Y, Kinoshita T. Human PIG-U and yeast Cdc91p are the fifth subunit of GPI transamidase that attaches GPI-anchors to proteins. Mol Biol Cell. 2003;14:1780-9.

17. Hou Y, Guo X, Cyprys P, Zhang Y, Bleckmann A, Cai L, Huang Q, Luo Y, Gu H, Dresselhaus T, Dong J, Qu LJ. Maternal ENODLs are required for pollen tube reception in Arabidopsis. Curr Biol. 2016;26:2343-50.

18. Huang $B Q$, Russell SD. Female germ unit - organization, isolation, and function. Int Rev Cytol Surv Cell Biol. 1992;140:233-93.

19. Ilgoutz SC, Zawadzki JL, Ralton JE, Mcconville MJ. Evidence that free GPI glycolipids are essential for growth of Leishmania mexicana. EMBO J. 1999; 18:2746-55.

20. Johnson MA, Harper JF, Palanivelu R. A fruitful journey: pollen tube navigation from germination to fertilization. Annu Rev Plant Biol. 2019;70: 809-37.

21. Johnson MA, Von Besser K, Zhou Q, Smith E, Aux G, Patton D, Levin JZ, Preuss D. Arabidopsis hapless mutations define essential gametophytic functions. Genetics. 2004;168:971-82.

22. Lalanne E, Honys D, Johnson A, Borner GH, Lilley KS, Dupree P, Grossniklaus U, Twell D. SETH1 and SETH2, two components of the glycosylphosphatidylinositol anchor biosynthetic pathway, are required for pollen germination and tube growth in Arabidopsis. Plant Cell. 2004;16:229-40

23. Leidich SD, Kostova Z, Latek RR, Costello LC, Drapp DA, Gray W, Fassler JS, Orlean P. Temperature-sensitive yeast Gpi anchoring mutants Gpi2 and Gpi3 are defective in the synthesis of N-Acetylglucosaminyl Phosphatidylinositol cloning of the Gp12 gene. J Biol Chem. 1995;270:13029-35.

24. Li C, Yeh FL, Cheung AY, Duan Q, Kita D, Liu MC, Maman J, Luu EJ, Wu BW, Gates L, Jalal M, Kwong A, Carpenter H, Wu HM.

Glycosylphosphatidylinositol-anchored proteins as chaperones and coreceptors for FERONIA receptor kinase signaling in Arabidopsis. Elife. 2015;4: e06587.

25. Liu X, Castro C, Wang Y, Noble J, Ponvert N, Bundy M, Hoel C, Shpak E, Palanivelu R. The role of LORELEl in pollen tube reception at the Interface of the Synergid cell and pollen tube requires the modified eight-cysteine motif and the receptor-like kinase FERONIA. Plant Cell. 2016;28:1035-52.

26. Mayfield JA, Preuss D. Rapid initiation of Arabidopsis pollination requires the oleosin-domain protein GRP17. Nat Cell Biol. 2000;2:128-30

27. Medof ME, Kinoshita T, Nussenzweig V. Inhibition of complement activation on the surface of cells after incorporation of decay-accelerating factor (DAF) into their membranes. J Exp Med. 1984:160:1558-78.

28. Medof ME, Nagarajan S, Tykocinski ML. Cell-surface engineering with GPIanchored proteins. FASEB J. 1996;10:574-86.

29. Medof ME, Walter El, Rutgers JL, Knowles DM, Nussenzweig V. Identification of the complement decay-accelerating factor (Daf) on epithelium and glandular cells and in body-fluids. J Exp Med. 1987;165:848-64.

30. Meyer U, Benghezal M, Imhof I, Conzelmann A. Active site determination of Gpi8p, a caspase-related enzyme required for glycosylphosphatidylinositol anchor addition to proteins. Biochemistry. 2000;39:3461-71.

31. Ohishi K, Inoue N, Kinoshita T. PIG-S and PIG-T, essential for GPI anchor attachment to proteins, form a complex with GAA1 and GPI8. EMBO J. 2001; 20:4088-98.

32. Orlean P, Menon AK. GPI anchoring of protein in yeast and mammalian cells, or: how we learned to stop worrying and love glycophospholipids. J Lipid Res. 2007;48:993-1011.

33. Pereira AM, Nobre MS, Pinto SC, Lopes AL, Costa ML, Masiero S, Coimbra S. "love is strong, and You're so sweet": JAGGER is essential for persistent Synergid degeneration and Polytubey block in Arabidopsis thaliana. Mol Plant. 2016;9:601-14.

34. Preuss D, Rhee SY, Davis RW. Tetrad analysis possible in Arabidopsis with mutation of the quartet (Qrt) genes. Science. 1994;264:1458-60.
35. Punwani JA, Rabiger DS, Drews GN. MYB98 positively regulates a battery of synergid-expressed genes encoding filiform apparatus-localized proteins. Plant Cell. 2007;19:2557-68.

36. Qin Y, Leydon AR, Manziello A, Pandey R, Mount D, Denic S, Vasic B, Johnson MA, Palanivelu R. Penetration of the stigma and style elicits a novel transcriptome in pollen tubes, pointing to genes critical for growth in a pistil. PLoS Genet. 2009;5:e1000621.

37. Qu LJ, Li L, Lan ZJ, Dresselhaus T. Peptide signalling during the pollen tube journey and double fertilization. J Exp Bot. 2015;66:5139-50.

38. Rosse WF. Phosphatidylinositol-linked proteins and paroxysmal-nocturnal Hemoglobinuria. Blood. 1990;75:1595-601.

39. Tsukamoto T, Qin Y, Huang YD, Dunatunga D, Palanivelu R. A role for LORELEl, a putative glycosylphosphatidylinositol-anchored protein, in Arabidopsis thaliana double fertilization and early seed development. Plant J. 2010:62:571-88.

40. Vaddepalli P, Fulton L, Wieland J, Wassmer K, Schaeffer M, Ranf S, Schneitz K. The cell wall-localized atypical beta-1,3 glucanase ZERZAUST controls tissue morphogenesis in Arabidopsis thaliana. Development. 2017;144:2259-69.

41. Wang L, Clarke LA, Eason RJ, Parker CC, Qi B, Scott R, Doughty J. PCP-B class pollen coat proteins are key regulators of the hydration checkpoint in Arabidopsis thaliana pollen-stigma interactions. New Phytol. 2017;213:764-77.

42. Zhan JP, Li GS, Ryu CH, Ma C, Zhang SS, Lloyd A, Hunter BG, Larkins BA Drews GN, Wang XF, Yadegari R. Opaque-2 regulates a complex gene network associated with cell differentiation and storage functions of maize endosperm. Plant Cell. 2018;30:2425-46.

43. Zhu YH, Fraering P, Vionnet C, Conzelmann A. Gpi17p does not stably interact with other subunits of glycosylphosphatidylinositol transamidase in Saccharomyces cerevisiae. Biochim Biophys Acta Mol Cell Biol Lipids. 2005; 1735:79-88.

\section{Publisher's Note}

Springer Nature remains neutral with regard to jurisdictional claims in published maps and institutional affiliations.

Ready to submit your research? Choose BMC and benefit from:

- fast, convenient online submission

- thorough peer review by experienced researchers in your field

- rapid publication on acceptance

- support for research data, including large and complex data types

- gold Open Access which fosters wider collaboration and increased citations

- maximum visibility for your research: over $100 \mathrm{M}$ website views per year

At $\mathrm{BMC}$, research is always in progress.

Learn more biomedcentral.com/submissions 